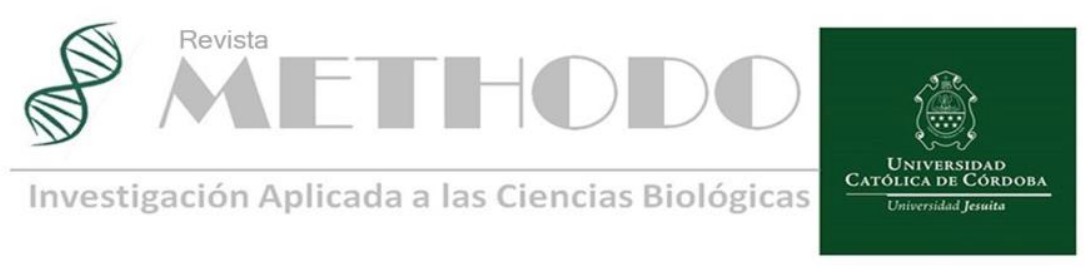

\title{
El juego del aprendizaje completo en la enseñanza de las ciencias $^{\text {a }}$
}

\section{The complete learning game in the teaching of sciences}

\author{
Horacio A. Ferreyra ${ }^{1}$. \\ 1Doctor y Licenciado en Educación (Universidad Católica de Córdoba), Subsecretario de Estado de Promoción de Igualdad y Calidad Educativa del Ministerio de \\ Educación de la provincia de Córdoba Secretario de Educación, Cultura e Innovación de la Municipalidad de Córdoba. Secretaria de Educación, Cultura e \\ Innovación de la Municipalidad de Córdoba. \\ Correspondencia: Horacio Ademar. Ferreyra; email: dr.horacio.ferreyra@gmail.com
}

a Una versión de este artículo sobre educación general básica fue publicado en la Revista Didasc@lia, Vol.7, núm.6 (2016).

\section{Resumen}

La presente comunicación está orientada a contribuir a la comprensión de algunas cuestiones vinculadas con la enseñanza y el aprendizaje de las ciencias en la Educación Superior, acudiendo a la categoría teórica de juego del aprendizaje completo de David Perkins, con el aporte de otros autores. Se plantean las razones y propósitos por los cuales se propone un cambio de paradigma, se analizan los principios del aprendizaje completo y se enuncian y ejemplifican algunas estrategias y condiciones de enseñanza pertinentes.

Palabras clave: enseñanza; aprendizaje; aprendizaje completo; enseñanza de las ciencias

\begin{abstract}
The present communication is aimed at contributing to the understanding of some issues related to the teaching and learning of science in Higher Education, going to the theoretical category of complete learning game by David Perkins, with the contribution of other authors. The reasons and purposes for which a paradigm shift is proposed are discussed, the principles of full learning are analyzed, and some relevant teaching strategies and conditions are stated and exemplified.
\end{abstract}

Keywords: teaching, learning, complete learning, science teaching.

\section{Introducción}

Las reflexiones y propuestas que a través de esta comunicación nos interesa compartir pretenden constituirse en un aporte orientado a contribuir a la comprensión -inteligente pero también sensiblede algunas cuestiones vinculadas con la enseñanza y el aprendizaje en las denominadas sociedades del conocimiento, como manifestación de nuestro compromiso con la educación en ciencias. Y decimos sociedades del conocimiento, en plural, porque entendemos que, en la actualidad, más que una única y gran sociedad, existen distintas "sociedades" interconectadas que interactúan en 
los procesos de construcción de saberes. Consideramos, entonces, que a partir de la diversidad y de la diferencia es posible construir mejores aprendizajes para todos.

Por otra parte, y en consonancia con esta mirada plural, nuestro aporte estará focalizado en el aprendizaje y la enseñanza de las ciencias. Y este plural es tal porque sostenemos que no hay que restringir la perspectiva a las ciencias naturales, sino involucrar también a las ciencias sociales, las humanidades y las disciplinas lógico-formales.

En este marco, lo que pretendemos compartir son algunas reflexiones en torno a la práctica educativa en las instituciones de educación superior. Asimismo, destacar especialmente la necesidad de un cambio de perspectiva en la enseñanza científica y tecnológica, en general, y establecer algunos principios para una educación más auténtica en las instituciones de Iberoamérica dedicadas a la formación en Ciencias de la Salud, en particular.

Se trata, entonces, de una práctica controvertida y compleja que requiere ser transformada. Como sostiene Sabalza ${ }^{1}$ :

"En una clase hay personas muy diferentes, cada una con su propia vida, sus diversas capacidades, su forma de estar, de integrarse a la dinámica del aprendizaje, etcétera. Están también las influencias del ambiente social y de la propia institución. Están los programas oficiales y el propio profesor. El docente también ha de abordar tareas tan distintas como impartir información y explicarla, atender el trabajo de cada uno de los estudiantes, juzgar su actividad y proporcionarles feedback, atender al grupo como tal y sintonizar emocionalmente con los distintos estados de las personas o sucesos de la clase, distribuir y operar con materiales y recursos. Cada decisión que se tome ha de ser congruente con este estado general de las cosas..." (p.209).

Efectivamente, la práctica educativa es compleja y a su vez controvertida porque en ella se conjuga la diversidad de sujetos -docentes, estudiantes-, las instituciones, los programas oficiales; $y$ se combinan diversas estrategias, actividades $\mathrm{y}$ recursos que se "ponen en acción en el aula", con el propósito de integrar la dimensión cognitiva y también la afectiva, en un proceso que reiteramos- es complejo y controvertido. Se refuerza así la idea de un docente como verdadero gestor de sus prácticas ${ }^{2}$ en contexto.

Por eso, el interrogante acerca de cómo aprende el que aprende y cómo enseña el que enseña en contextos de la educación superior será la pregunta que atravesará nuestras reflexiones.

\section{Desarrollo}

Partimos esencialmente de un problema que confronta dos puntos de vista: el del mundo que cambia aceleradamente y el de la institución educativa de nivel superior, que parece permanecer idéntica a sí misma a través de los años. En el marco de esta tensión es que hoy vemos a la universidad como un lugar donde priman todavía los fragmentos, los segmentos, y donde cuesta poder mirar la integralidad; donde muchas veces no nos posibilitamos, tanto docentes como estudiantes, "jugar el juego completo del aprendizaje", como propone Perkins ${ }^{3}$.

Si para explicarlo acudimos a una analogía con el fútbol, advertimos que jugar el juego completo del aprendizaje implica, desde el inicio, poder tener una pelota, correr tras ella $\mathrm{y}$, particularmente, poder ir aprendiendo a medida que se va "haciendo". Esto no impide que, en cierto momento, tengamos que "detener el partido" para enseñar a patear un penal, un córner, o la estrategia de una determinada jugada... pero desde el comienzo debemos posibilitar al estudiante "jugar el juego completo" del aprendizaje. Esto demanda pensar en versiones para principiantes, en versiones para novatos, que podamos ir complejizando a medida que vayan avanzado las trayectorias educativas en el nivel superior.

En este punto, resulta importante referir algunas características y/o aspectos del nivel superior de educación que hoy están poniendo frenos a esta idea de poder jugar el juego completo del aprendizaje. El primero de ellos tiene que ver con la tendencia a abordar los objetos de aprendizaje de manera atomizada, de manera gradual, aprendiendo primero los componentes particulares sin integrarlos en el todo. A esto, se suma la idea de que primero hay que teorizar y después hacer. En consecuencia, son pocas las experiencias que les permiten a los estudiantes jugar desde el inicio el juego completo del aprendizaje en torno a una determinada actividad, en torno a una determinada acción porque -en términos de Perkins ${ }^{3}$ - la elementitis y la acerquitis nos atrapan, nos cierran a la posibilidad de esa actividad holística, de esa mirada amplia que nos permita ver que se "aprende haciendo" con ciencia y con conciencia.

\section{Un cambio de paradigma}

A los docentes del nivel superior de educación, investigadores y tecnólogos nos cuesta mucho poder enfrentar la aventura intelectual. A veces, la queremos hacer paso a paso y acabadamente, y nos rehusamos a la posibilidad de emprender, de considerar al otro como sujeto, de buscar, de tamizar, de sintetizar, de buscar nuevos horizontes. En este sentido, cabe preguntarnos qué podemos hacer para que los contenidos sean más accesibles

Revista Methodo: Investigación Aplicada a las Ciencias Biológicas. Universidad Católica de Córdoba. Jacinto Ríos 571 Bo Gral. Paz. X5004FXS. Córdoba. Argentina. Tel.: (54) 3514517299 / Correo: methodo@ucc.edu.ar / Web: methodo.ucc.edu.ar | ARTICULO DE REVISION Rev. Methodo 2020;5(1):04-08. 
a nuestros estudiantes y, correlativamente, cuáles son las prácticas que pueden motivar, que pueden estimular para que realmente nuestros estudiantes no sólo puedan conocer, sino que, a medida que lo hacen, puedan comprender.

Lo que se requiere, entonces, es el cambio de un paradigma con centro en quien enseña, a otro centrado en quien aprende, sin menoscabo de la enseñanza ${ }^{4}$. Esta breve referencia a los paradigmas educativos nos conduce a reflexionar acerca de la necesidad de plantearnos, en cada momento de la enseñanza de las Ciencias de la Salud, tres grandes preguntas. Cuando nos formulamos la primera $-i \mathrm{a}$ quién enseñar? - aparecen los participantes; y en relación con ellos, la clave es dejar de mirar el déficit para pasar fundamentalmente a pensar en los sujetos como potencias, como personas distintas que piensan, sienten y hacen de manera diferente.

Pero a esta primera pregunta hay que sumarle otra, que está vinculada no sólo con el que enseña, sino fundamentalmente con el para qué enseña: ¿qué es lo que yo, en mi rol docente, tengo que enseñarles a mis estudiantes porque si yo no sé los enseño en este curso, en este año, no lo podrán aprender durante la vida? Esta pregunta es estelar porque nos lleva a focalizarnos, a centrarnos en aquello que realmente tenemos que enseñar en la educación superior porque-siguiendo el razonamiento- si yo no lo enseño, puedo estar perjudicando el desarrollo integral de este futuro profesional -en nuestro caso particular- de la salud, con el cual comparto la aventura se enseñar. Se trata, en suma, de un interrogante que nos conduce a no poner sólo el acento en la eficacia, en la eficiencia, en las evaluaciones y sus resultados, sino a mirar la pertinencia y la relevancia de los saberes en cada uno de los contextos.

La tercera pregunta a considerar desde la enseñanza en general y desde la de las ciencias de la salud en particular, se vincula al cómo enseñar, es decir, a esas estrategias tanto reproductoras como productoras y transformadoras que me permiten acercar y construir el conocimiento con otros. En esto, la propuesta -inspirada prioritariamente en Perkins ${ }^{3,5,6}$ y en Gardner ${ }^{7,8,9,10}$ es pensar algunas estrategias, algunas acciones para construir mejor en torno a los procesos de enseñanza y aprendizaje.

En este marco y para fundamentar la propuesta, lo que pretendemos compartir no es ninguna teoría, sino particularmente un enfoque -que podríamos llamar contemporáneo- que recupera una acción integradora, que nos posiciona no en el o sino fundamentalmente en él y: no es constructivismo versus conductismo, sino constructivismo, conductismo, conectivismo y toda esta perspectiva que de una manera integral permite comprender cómo aprende el que aprende y cómo enseña el que enseña.

Partimos de la idea de que cualquier asignatura, como decía Bruner ${ }^{11,12,13}$, puede ser enseñada de forma efectiva a través de diferentes caminos y formas cognitivas a todos los sujetos en cualquier estado de su desarrollo; es decir, se trata de ir complejizando la actividad educativa a medida que se van aumentando los niveles de formación. Esto implica, como Bruner lo decía, pensar en un currículum en espiral, con mayores niveles de complejidad y distintos niveles de profundidad.

Por y para un aprendizaje completo

Para poder reflexionar en torno a cómo aprende el que aprende y cómo enseña el que enseña en contextos educativos formales, proponemos tomar en cuenta los principios que enuncia Perkins3: el planteamiento de actividades holísticas que les posibiliten a los estudiantes el aprendizaje completo, lograr "que valga la pena desarrollar esa actividad", es decir, generar un vínculo con la motivación; trabajar las "partes difíciles", posibilitar la transferencia, descubrir el "juego oculto" del aprendizaje, aprender del equipo y de los otros equipos y, fundamentalmente, aprender a aprender.

A continuación, vamos a ir considerando estrategias de enseñanza en las que las verdades absolutas dan lugar a las verdades relativas, el docente deja de ser transmisor para pasar a ser mediador o facilitador, el estudiante deja de ser considerado un sujeto que no sabe nada, para constituirse en el protagonista. Estas estrategias ponen de manifiesto que aprender no es repetir sino, fundamentalmente, transferir, y que el contexto deja de ser un marco estático para hacerse dinámico, resignificado en situación.

Nuestra primera estrategia de enseñanza tiene que ver con esta intención de jugar el juego completo del aprendizaje; aquí el docente plantea propuestas como: En esta localidad se han registrado personas con intoxicación por plomo... ¿Qué hacemos como personal de salud de esta unidad sanitaria? Desde el inicio de la enseñanza y el aprendizaje de un contenido, el profesor pone el acento en el hacer y, simultáneamente, en la movilización de los recursos cognitivos de los estudiantes y en la provisión de nuevos contenidos y de materiales teóricos. Para estos estudiantes, la tarea no implica solamente "hacer cosas", sino repensar lo hecho y, fundamentalmente, poder explicar y justificar por qué lo hicieron de esa manera, disponiendo de la teoría.

La invitación es a pensar, desde las instituciones educativas de nivel superior dedicadas a las ciencias de la salud, en aprendizajes basados en problemas, en aprendizajes basados en proyectos, como así también en verdaderos estudios de

Revista Methodo: Investigación Aplicada a las Ciencias Biológicas. Universidad Católica de Córdoba. Jacinto Ríos 571 Bo Gral. Paz. X5004FXS. Córdoba. Argentina. Tel.: (54) 3514517299 / Correo: methodo@ucc.edu.ar / Web: methodo.ucc.edu.ar | ARTICULO DE REVISION Rev. Methodo 2020;5(1):04-08. 
campo. Pero para que realmente "el juego completo" del aprendizaje se dé en cada uno de los contextos, hace falta tener en cuenta algunos principios fundamentales:

$\square$ Un ritmo de trabajo dinámico que permita a los estudiantes y al docente percibir que no están perdiendo tiempo.

$\neg$ Poner el foco en el problema para no alejarse del tema-eje, es decir, sostener la centralidad del objetivo.

Plantear el desafío como tolerable, esto es, un desafío que los estudiantes sientan que puedan enfrentar.

$\square$ Comprender que los procesos de consolidación suponen repaso, construcción de esquemas, revisión y balances, para que realmente se puedan ir construyendo las ideas.

Otro de los principios que Perkins ${ }^{3}$ enuncia es la necesidad de que la actividad sea motivadora ("lograr que valga la pena jugar el juego"), para lo cual tiene que poder vincular el saber con la realidad, la investigación con la acción y la teoría con la práctica. En esto, los tópicos generativos, los "enganches" narrativos, son claves, son pertinentes y relevantes. Preguntar a los estudiantes qué harían frente a los resultados de estos exámenes clínicos, qué decisión tomarían respecto de estos datos provistos por un paciente pueden ser caminos que nos conduzcan a buscar más allá de las tradicionales unidades didácticas temáticas para la enseñanza de las ciencias de la salud, despertando en los estudiantes el espíritu de curiosidad, de indagación, de búsqueda.

Frente a las situaciones de enseñanza para el aprendizaje que tradicionalmente plantea la educación superior, acontecen -de parte de los estudiantes- diversas formas de resistencia pasiva (desear no estar presentes, ansiar liberarse "para ir a hacer otra cosa", evadirse, ausentarse, entre otras), que nos deben preocupar y nos deben ocupar para poder realmente activar la motivación en cada una de nuestras prácticas.

Hace falta, además, -dice Perkins ${ }^{3}$ - "trabajar las partes difíciles", y esto significa no trabajar aquello que sabemos que nuestros estudiantes están en condiciones de abordar por sí mismos, sino dedicarnos a las cuestiones que pueden ser más difíciles para ellos. El docente tiene que ser capaz de anticipar esas "partes difíciles" a fin de poder focalizar los procesos de enseñanza y de aprendizaje en ellas a través de actividades específicas previamente diseñadas. En todo esto tiene un lugar muy importante la evaluación, pero con énfasis en los procesos evaluativos, en los logros de los estudiantes, en vez de apresurarnos a medir resultados a través del test o la prueba.

También nos invita Perkins ${ }^{3}$ a que llevemos a nuestros estudiantes a "jugar de visitantes", es decir, a transferir lo aprendido a otras situaciones para seguir aprendiendo durante toda la vida. Sin embargo, para que la transferencia se dé hacen falta algunos ingredientes previos, tales como permitirles a nuestros estudiantes que expresen libremente sus ideas, que puedan comunicarse y, fundamentalmente, que puedan hacer y disponer de lo aprendido para abordar nuevas situaciones. Cuando se aprende, se manifiesta "el juego oculto" del aprendizaje, aquellos saberes que no están puestos en evidencia en nuestras prácticas educativas, pero que son claves para seguir aprendiendo durante toda la vida. Lo importante es no quedarnos con la "cáscara de la naranja", sino profundizar en su interior: en los gajos que la integran, en sus sabores y sinsabores para que realmente el aprendizaje sea significativo, pertinente y relevante.

Aprender del equipo y de los otros equipos y, fundamentalmente, aprender a aprender son los otros dos principios relevantes. El primero, se relaciona con la posibilidad de aprender de otros para poder proyectarse $y$ crecer en el conocimiento. El segundo, convoca al docente -sin renunciar a la guía y el acompañamiento- a dar el lugar de conductores a los estudiantes, para que dejen de ocupar siempre el lugar de "acompañantes" y nos puedan (de) mostrar que, efectivamente, están aprendiendo. Se trata de forjar, como se expresa en la Declaración ${ }^{14}$ emanada de la III Conferencia Regional de Educación Superior para América Latina y el Caribe, "comunidades de trabajo donde el anhelo de aprender y la construcción dialógica y crítica del saber entre docentes y estudiantes sea la norma".

\section{Reflexiones finales}

En las actuales sociedades del conocimiento hace falta potenciar el desarrollo de la inteligencia y, para eso, como dice Gardner ${ }^{7}$, no se trata de pensar sólo en una inteligencia láser, superespecializada, sino en la importancia que adquiere una inteligencia faro, esa que va a habilitar miradas holísticas, que va a permitir comprender la situación, asumir compromisos y desarrollar una construcción efectiva. Para poder comprender procesos de salud no es suficiente conocer la diversidad de las ciencias (naturales, sociales, lógico-formales) y las humanidades; la "mente disciplinada" no alcanza, sino que se precisa una "mente interdisciplinada" en la que los saberes puedan conectarse. Para ello, tenemos que poner el acento en enseñar a nuestros estudiantes a captar, a procesar y a comunicar información, esto es, tener una mente sintetizadora. Hoy, la información

Revista Methodo: Investigación Aplicada a las Ciencias Biológicas. Universidad Católica de Córdoba Jacinto Ríos 571 Bo Gral. Paz. X5004FXS. Córdoba. Argentina. Tel.: (54) 3514517299 / Correo: methodo@ucc.edu.ar / Web: methodo.ucc.edu.ar | ARTICULO DE REVISION Rev. Methodo 2020;5(1):04-08. 
nos invade; por lo tanto, hace falta a partir de ella generar conocimiento.

Por otro lado, es necesario comenzar a respetar la diversidad y en esa diversidad las diferencias, para poder trabajar asumiendo en cada uno de nuestros proyectos un compromiso ético; advertir que del otro lado está ese otro con quien construir saberes para poder comprender la realidad, no solamente de manera inteligente sino fundamentalmente de manera sensible. La invitación es, entonces, a no limitarnos a distribuir conocimientos en las aulas de la educación superior que forma en ciencias de la salud, sino a aplicar verdaderas políticas de reconocimiento. Reconozcamos a ese otro, y a partir de ese otro enseñemos.

Finalmente, compartimos con Perkins ${ }^{3}$ una frase para seguir pensado: "el mundo sería un lugar mejor si la gente lograra una mediocridad activa, en vez de una erudición pasiva". Nuestra apuesta es, entonces, a dejar ese lugar de pasividad de lo intelectual, para sumarnos a la aventura intelectual de hacer ciencia con conciencia, asumir un compromiso ético y lograr una construcción que mejore nuestras prácticas educativas.

\section{Bibliografía}

1. Sabalza, M.A. Organización de las estrategias de enseñanza. En: Diseño y desarrollo curricular. Madrid: Narcea; 1997. p.149-234.

2. ITSM, Instituto Tecnológico Superior de Monterrey. El profesor como gestor de su práctica docente. En Manual de la Práctica Docente con base en la misión para el 2005. Monterrey: ITESM; 2001. Recuperado a partir de http://www.sistema.itesm.mx/va/DraMarisa/ Modulo2.html

3. Perkins, D. El aprendizaje pleno. Principios de la enseñanza para transformar la educación. Buenos Aires: Paidós; 2010. 296 p.
4. Bain, K. Lo que hacen los mejores profesores de la universidad. Barcelona: Publicaciones de la Universidad de Valencia; 2007. 239 p.

5. Perkins, D. La escuela inteligente: del adiestramiento de la memoria a la educación de la mente. Barcelona: Gedisa; 1995. 262 p.

6. Perkins, D. Enseñar para comprender en el siglo XXI. Conferencia. II Simposio Internacional de Enseñanza para la Comprensión en Educación Superior. Universidad Nacional de Villa María; Córdoba, Argentina, septiembre 2012.

7. Gardner, H. Inteligencias múltiples: la teoría en la práctica. Barcelona: Paidós; 1998. 383 p.

8. Gardner, H. La inteligencia reformulada. Barcelona: Paidós; 2001.272 p.

9. Gardner, H. Las cinco mentes del futuro. Buenos Aires: Paidós; 2008. 258 p.

10. Gardner, H. La nueva ciencia de la mente. Barcelona: Paidós; 2011. 448 p.

11. Bruner, J. Hacia una teoría de la instrucción. México: Hispanoamericana; 1969. 234 p.

12. Bruner, J. Desarrollo cognitivo y educación. Madrid: Morata; 1988.338 p.

13. Bruner, J. La educación, puerta de la cultura. Madrid: Visor; 1997. 216. 224 p.

14. CRES. Declaración III Conferencia Regional de Educación Superior para América Latina y el Caribe, Córdoba, Argentina, 14 de junio de 2018.

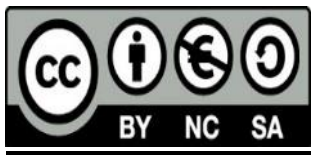

